\title{
High Resolution OFDM Channel Estimation with Low Speed ADC using Compressive Sensing
}

\author{
Jia (Jasmine) Meng ${ }^{1}$, Yingying Li ${ }^{1,2}$, Nam Nguyen ${ }^{1}$, Wotao Yin ${ }^{2}$ and Zhu Han ${ }^{1}$ \\ ${ }^{1}$ Department of Electrical and Computer Engineering, University of Houston \\ ${ }^{2}$ Department of Computational and Applied Mathematics, Rice University
}

\begin{abstract}
Orthogonal frequency division multiplexing (OFDM) is a technique that will prevail in the next generation wireless communication. Channel estimation is one of the key challenges in an OFDM system. In this paper, we formulate OFDM channel estimation as a compressive sensing problem, which takes advantage of the sparsity of the channel impulse response and reduces the number of probing measurements, which in turn reduces the ADC speed needed for channel estimation. Specifically, we propose sending out pilots with random phases in order to "spread out" the sparse taps in the impulse response over the uniformly downsampled measurements at the low speed receiver ADC, so that the impulse response can still be recovered by sparse optimization. This contribution leads to high resolution channel estimation with low speed ADCs, distinguishing this paper from the existing attempts of OFDM channel estimation. We also propose a novel estimator that performs better than the commonly used $\ell_{1}$ minimization. Specifically, it significantly reduces estimation error by combing $\ell_{1}$ minimization with iterative support detection and limited-support least-squares. While letting the receiver $A D C$ run at a speed as low as $1 / 16$ of the speed of the transmitter DAC, we simulated various numbers of multipaths and different measurement SNRs. The proposed system has channel estimation resolution as high as the system equipped with the high speed ADCs, and the proposed algorithm provides additional $6 \mathrm{~dB}$ gain for signal to noise ratio.
\end{abstract}

\section{INTRODUCTION}

Orthogonal frequency division multiplexing (OFDM) has been widely applied in wireless communication systems, because it transmits at a high rate, achieves high bandwidth efficiency, and is robust to multipath fading and delay [1]. OFDM applications can be found in digital television and audio broadcasting, wireless networking, and broadband internet access. Current OFDM based WLAN standards (such as IEEE $802.11 \mathrm{a} / \mathrm{g}$ ) use variations of QAM schemes for subcarrier modulations which require a coherent detection at the OFDM receiver and consequently requires an accurate (or near accurate) estimation of Channel State Information (CSI). The structure of OFDM signal makes it difficult to balance complexity and performance in channel estimation. The design principles for channel estimators are to reduce the computational complexity and bandwidth overhead while maintaining sufficient estimation accuracy.

Some channel estimation schemes proposed in literature are based on pilots, which form the reference signal used by both the transmitter and the receiver. This approach has two main challenges: (i) the design of pilots; and (ii) the design of an efficient estimation algorithm (i.e., the estimator).
There is a tradeoff between the spectrum efficiency and the channel estimation accuracy. Most of the existing pilotassisted OFDM channel estimation schemes rely on the use of a large number of pilots to increase to estimation accuracy; the spectral efficiency is therefore reduced. For example, there are approaches based on time-multiplexed pilot, frequencymultiplexed pilot, and scattered pilot [2], all achieving higher estimation accuracy at the price of using more pilots. There have been attempts to reduce the number of pilots, i.e. J. Byun et al. in [3]. However, the solutions generally require extra "test signal" for channel pre-estimation. By sending out "test signal", they try to find out how many pilots are needed by firstly inserting a relatively small number of pilots and then, based on the results of the "test", the number of pilots are decided. Therefore, there is no guaranteed overall reduction of pilots insertion.

As a sensing problem, OFDM channel estimation can benefit from the emerging technique of compressive sensing (CS), which acquires and reconstructs a signal from fewer samples than what is dictated by the Nyquist-Shannon sampling theorem, mainly by utilizing the signal's sparse or compressible property. The field has exploded since the pioneering work by Donoho [4] and Candes, Romberg and Tao [5]. The main idea is to encode a sparse signal by taking its "incoherent" linear projections and recover the signal through algorithms such as $\ell_{1}$ minimization. To maximize the benefits of CS for OFDM channel estimation, one shall skillfully perform the CS encoding and decoding steps, which are precisely the two focuses of this paper: the designs of pilots and estimator, respectively.

Contributions: CS has been applied to channel estimation in [13-16], which are reviewed in subsection III-E below. For OFDM channel estimation, there are papers [6-9], to which our work differs in various ways as follows. We skillfully design CS encoding and decoding strategies for OFDM channel estimation. Compared to existing work, we are able to obtain channel response in much higher resolutions and from much fewer pilots (thus taking much shorter time). This is achieved by designing pilots with uniform random phases and using a novel estimator. The pilot design preserves the information of high-resolution channel response during aggressive uniform down-sampling, which means that receiver $\mathrm{ADC}$ can run at a much lower speed. The estimator is tailored for OFDM channel response; in particular, instead of the generic $\ell_{1}$ minimization, iterative support detection (ISD) [17] 
and limited-support least-squares are adopted in order to take advantage of the characteristics of channel response. The resulting algorithm is very simple and performs better.

The rest of this paper is organized as follows: Section II reviews the general OFDM system model and sets up the channel estimation formulation. Section III relates channel estimation to CS and presents the proposed pilot design. In Section IV, the estimator based on iterative support detection and limited-support least-squares are introduced. Section V gives the simulation results. Finally, Section VI concludes this work.

\section{OFDM SYSTEM MODEL}

A baseband OFDM system is shown in Figure 1. In this system, the modulated signal in the frequency domain, represented by $\mathbf{X}(k), k \in[1, N]$, is inserted with pilot signal and guard band, and then an $N$-point IDFT transforms the signal into the time domain, denoted by $x(n), n \in[1, N]$, where a cyclic extension of time length $T_{G}$ is added to avoid inter-symbol and inter-subcarrier interferences. The resulting time series data is converted by a digital-to-analog converter (DAC) with a clock speed of $1 / T_{S} \mathrm{~Hz}$ into an analog signal for transmission. We assume that the channel response comprises $P$ propagation paths, which can be modeled by a time-domain complex-baseband vector with $P$ taps:

$$
h(n)=\sum_{p=1}^{P} \alpha_{p} \delta\left(n-\tau_{p} T_{S}\right), n=1, \ldots, N,
$$

where $\alpha_{p}$ is a complex multipath component and $\tau_{p}$ is the multipath delay $\left(0 \leq \tau_{p} T_{S} \leq T_{G}\right)$. Since $T_{G}$ is less than the OFDM symbol durations, the nonzero channel response concentrates at the beginning, which translates to $h=$ $\left[h_{1}, h_{2}, \ldots, h_{\tilde{N}}, 0, \ldots, 0\right]$, i.e., only the first $\tilde{N}$ components of $h$ can possibly take nonzero values and $\tilde{N}<N$. Assuming that interferences are eliminated, what arrives at the receiver is the convolution of the transmitted signal and the channel response plus noise, denoted by $z(n)$ given by

$$
z=x \otimes h+\xi,
$$

where $\otimes$ denotes convolution and $\xi(n), n \in[1, N]$ denotes the sampled AWGN noise. Passing through the analog-to-digital converter (ADC), $z(n), n \in[1, N]$ is sampled as $y(m), m \in$ $[1, M]$, and the cyclic prefix (CP) is removed. Traditional OFDM channel estimation schemes assume $M=N$. If $M<N$, then $y$ is a downsample of $z$. An $M$-point DFT converts $y$ to $\mathbf{Y}(k), k \in[1, M]$, where the guard band and pilot signal will be removed.

For pilot assisted OFDM channel estimation, we shall design the pilots $\mathbf{X}$ (and thus $x$ ) and recover $h$ from the measurements $\mathbf{Y}$ (or, equivalently $y$ ).

\section{Compressive SENSING OFDM Channel ESTIMATION}

\section{A. Motivations}

CS, which will be reviewed in the next subsection, allows sparse signals to be recovered from very few measurements,

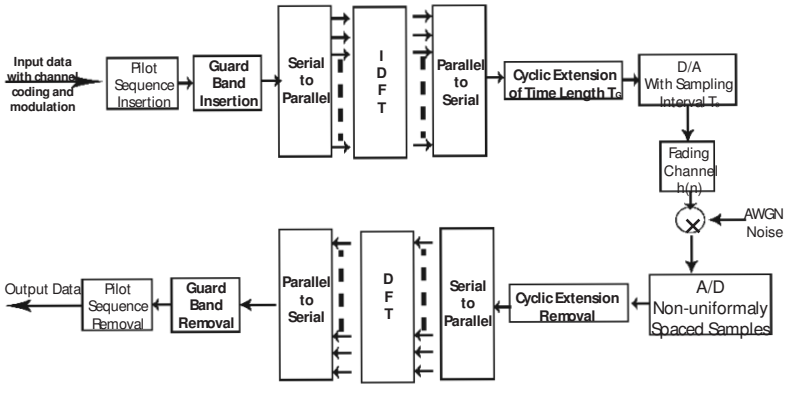

Fig. 1. Baseband OFDM System

which translates to slower sampling rates and shorter sensing times. Because the channel impulse response $h$ is very sparse, we are motivated to apply CS to recover $h$ by using a reduced number of pilots so that the estimation becomes much faster. Furthermore, in a sharp contrast to conventional OFDM channel estimation in which ADC and DAC run at the same sampling rate, we can obtain a higher-resolution $h$ by increasing the sampling rate of only the transmitter DAC, or we can reduce the receiver ADC speed which often defines the system cost. In other words, we have $N>M$. The rest of this section reviews CS and introduces our proposed approach for OFDM channel estimation.

\section{B. CS Background}

CS theories [4], [10], [11] state that a $S$-sparse signal ${ }^{1} h$ can be stably recovered from linear measurements $y=\Phi h+\xi$, where $\Phi$ is a certain matrix with $M$ rows and $N$ columns, $M<N$, by minimizing the $\ell_{1}$-norm of $h$. Classical CS often assumes that $\Phi$, after scaling, satisfies the restricted isometry property (RIP) $(1-\delta)\|h\|_{2}^{2} \leq\|\Phi h\|_{2}^{2} \leq(1+\delta)\|h\|_{2}^{2}$ for all $S$-sparse $h$, where $\delta>0$ is the RIP parameter. The RIP is satisfied with high probability by a large class of random matrices, e.g., those with entries independently sampled from a subgaussian distribution. By minimizing the $\ell_{1}$-norm, one can stably recover $h$ as long as $M \geq O(S \log N)$.

The classical random sensing matrices are not admissible in OFDM channel estimation because the channel response $h$ is not directly multiplied by a random matrix; instead, as describe in Section II, $h$ is first convoluted with $x$, the noise is added, and then the received signal $z$ is uniformly down-sampled to $y$. Because convolution is a circulant linear operator, we can present this process by $y=\downarrow_{\Omega} z=\downarrow_{\Omega}(C h+\xi)$, where the sensing matrix $C$ is the full circulant (convolution) matrix determined by $x$, and $\downarrow_{\Omega}$ denotes the uniform down sampling at points in $\Omega=\{1,1+N / M, \ldots, N-N / M+1\}$. As is widely perceived, CS favors fully random matrices, which admit stable recovery from fewest measurements (in terms of order of magnitude), but $C$ is structured and thus much less "random". This factor seemingly suggests that $C$ would be not favored by CS. Nevertheless, carefully designed circulant matrices can deliver the same optimal CS performance.

\footnotetext{
${ }^{1}$ In our case, $S$ is equal to $P$, the number of non-zero taps in (1).
} 


\section{Pilot with Random Phases}

To design the sensing matrix $C$, we propose to generate pilots $\mathbf{X}$ in either one of the following two ways: (i) the real and imaginary parts of $\mathbf{X}(k)$ are sampled independently from a Gaussian distribution, $k=1, \ldots, N$; (ii) (same as [15]) $\mathbf{X}(k), k=1, \ldots, N$, has independent random phases but a uniform amplitude. Note that $\mathbf{X}(k)$ of type (i) also have independent random phases. Let $F$ denote the discrete Fourier transform. Following from the convolution theorem $x \otimes h=F^{-1}(F(x) \cdot F(h))$ and $x=F^{-1}(\mathbf{X})$, we have $x \otimes h=F^{-1} \operatorname{diag}(\mathbf{X}) F h$, so the measurements $y$ can be written as

$$
y=\downarrow_{\Omega}(C h+\xi)=\downarrow_{\Omega}\left(F^{-1} \operatorname{diag}(\mathbf{X}) F h+\xi\right) .
$$

Let us explain intuitively why (3) is an effective encoding scheme for a sparse vector $h$. First, it is commonly known that $F h$ is non-sparse and its mass is somewhat evenly spread over all its components. The random phases of $\mathbf{X}$ by design are of critical importance. They "scramble" $F h$ component wisely and break the delicate relationships among $F h$ 's components; as a result, in contrast to the sparse $F^{-1} F h=h$, $F^{-1} \operatorname{diag}(\mathbf{X}) F h$ is not sparse at all. Furthermore, $\mathbf{X}$ has a random-spreading effect. Due to a phenomenon called concentration of measure [12], the mass of $C h$ spreads over its components in a way that, with high probability, the information of $h$ is preserved by down sampling of a size essentially linear in $P-$ the sparsity of $h$ (whether or not the down-sampling is equally spaced, i.e., uniform). Up to a $\log$ factor, the down sampled measurements permit stable $\ell_{1}$ recovery. Both types (i) and (ii) of $\mathbf{X}$ have similar encoding strength, but $\mathbf{X}$ of type (ii) gives an orthogonal $C$, i.e., $C^{*} C=I$, so $x \otimes h$ transforms $h$ into a random orthobasis. Such orthogonality results in multiple benefits such as faster convergence of our recovery algorithm. Due to the page limitation, we omit rigorous mathematical analysis of (3) and its guaranteed recovery.

Note that the proposed sampling $\downarrow_{\Omega}\left(F^{-1} \operatorname{diag}(\mathbf{X}) F\right)$ is very different from partial Fourier sampling $\downarrow_{\Omega} F$. The latter requires a random $\Omega$ to avoid the aliasing artifacts in the recovery but the former, with randomly-phased $\mathbf{X}$, permits both random and uniform $\Omega$. Below we numerically demonstrate its encoding efficiency.

\section{Numerical Evidence of Effective Random Convolution}

CS performance is evaluated by the number of measurements required for stable recovery. To compare the proposed sensing schemes with the well-established Gaussian random sensing, we conduct numerical simulations and show its results in Figure 2. We compare three types of CS encoding matrices: the i.i.d. Gaussian random complex matrix, and the circulant random complex matrices corresponding to $\mathbf{X}$ of types (i) and (ii) above, respectively. In addition, $\ell_{1}$ minimization is compared to our proposed algorithm CS-OFDM, which is detailed in the next section. The simulations results show that the random convolutions of both types perform as well as the Gaussian random sensing matrix under $\ell_{1}$ minimization, and

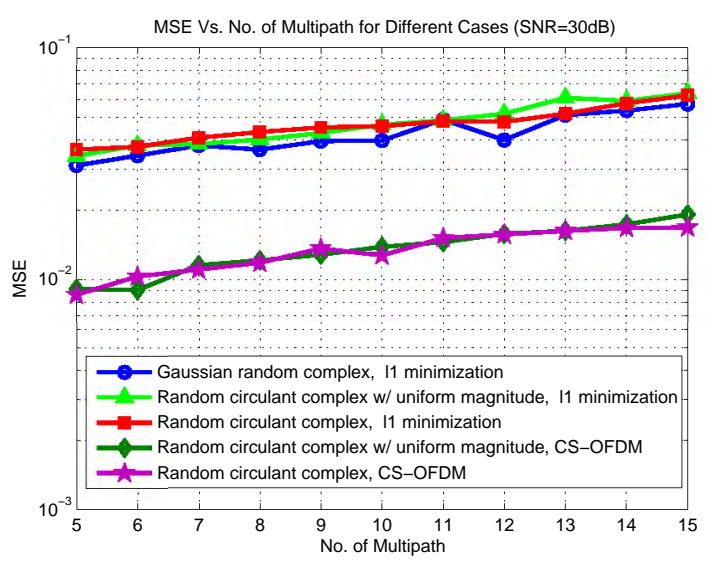

Fig. 2. MSE Vs. No. of Multipath for Different Cases $(\mathrm{SNR}=30 \mathrm{~dB})$.

our algorithm CS-OFDM further improves the performance by half of a magnitude.

\section{E. Relationship to Existing Results}

1) Random Convolution CS: Random convolution has been used and proved to be an effective way of taking CS measurements that allow the signal to be recovered using $\ell_{1}$ minimization. In [13], Toeplitz ${ }^{2}$ measurement matrices are constructed with i.i.d random row 1 (the same as type (i)) but with only \pm 1 or $\{-1,0,1\}$; their down sampling effectively takes the first $M$ rows; and the number of measurements needed for stable $\ell_{1}$ recovery is shown as $M \geq O\left(S^{3} \cdot \log N / S\right)$. [14] uses a "partial" Toeplits matrix, with i.i.d. Bernoulli or Gaussian row 1, for sparse channel estimation where the down sampling effectively also takes the first $M$ rows. Their scheme requires $M \geq O\left(S^{2} \cdot \log N\right)$ for stable $\ell_{1}$ recovery. In [15], random convolution of type (ii) above with either random downsampling or random demodulation is proposed and studied. It is shown that the resulting measurement matrix is incoherent with any given sparse basis with high probability and $\ell_{1}$ recovery is stable given $M \geq O\left(S \cdot \log N+\log ^{3} N\right)$. Our proposed type (ii) is motivated by [15]. On the other hand, no existing work proposes uniform down-sampling or shows its recovery guarantees. In addition, most existing analysis is limited to real-valued matrices and signals.

2) CS-based Channel Estimation: Our work is closely related to [14] and [16]. In [14], i.i.d. Bernoulli or Gaussian vector is used as training sequence, and downsample is carried out by taking only the first $M$ rows, while channel estimation is obtained as a solution to the Dantzig selector. In [16], MIMO channels are estimated by activating all sources simultaneously. The receivers measure the cumulative response, which consists of random convolutions between multiple pairs of source signals and channel responses. Their goal is to reduce the channel estimation time. $\ell_{1}$ minimization is used to recover channel response.

Our current work is limited to estimating a signal $h$-vector. While our work is based on similar random convolution techniques, we have proposed to use a pair of high-speed

\footnotetext{
${ }^{2}$ which is slightly more general than circulant.
} 
source and low-speed receiver for the novel goal of high resolution channel estimation. Furthermore, we apply a novel algorithm for the channel response recovery based on iterative support detection and limited-support least-squares, which is described in details in Section IV below.

\section{NumericAl Algorithm}

\section{A. Problem Formulation}

As a result of rapid decaying of wireless channels, $P$ - the number of significant multipaths - is small, so the channel response $h$ is a highly sparse signal. Recall that the non-zero components of $h$ only appear in the first $\tilde{N}$ components. We shall recover a sparse high-resolution signal $h$ with a constraint from the measurements $y$ at a lower resolution of $M$. We define operation $|\cdot|$ as the amplitude of a complex number, $\|h\|_{0}$ as the total number of nonzero entries in $|h|$ and $\|h\|_{1}=$ $\sum_{i}\left|h_{i}\right|$. The corresponding model is

$$
\min _{h \in \mathbb{R}^{N}}\|h\|_{0} \text {, s.t. } \phi h=y \text { and } h_{i}=0 \forall i>\tilde{N},
$$

where $\phi$ denotes $\downarrow_{\Omega} C$ in (3), the submatrix of $C$ formed by its rows corresponding to the down-sampling points in $\Omega$. Generally speaking, problem (4) is NP-hard and is impossible to solve even for moderate $N$. A common alternative is its $\ell_{1}$ relaxation model with the same constraints.

$$
\min _{h \in \mathbb{R}^{N}}\|h\|_{1} \text {, s.t. } \phi h=y \text { and } h_{i}=0 \forall i>\tilde{N},
$$

which is convex and has polynomial time algorithms. If $y$ has no noise, both (4) and (5) can recover $h$ exactly given enough measurements, but (5) requires more measurements than (4).

\section{B. Algorithm}

Instead of using a generic algorithm for (5), we design an algorithm to exploit the OFDM system features, including the special structure of $h$ and noisy measurements $y$. At the same time, we maintain its simplicity to achieve low complexity and match with easy hardware implementation.

First of all, we can simply collaborate two constraints into one by letting the variables be $\tilde{h}=\left[h_{1}, h_{2}, \ldots, h_{\tilde{N}}\right]$ and dropping the rest components of $h$. Let $\tilde{\phi}$ be the matrix formed by first $\tilde{N}$ columns of $\phi$. Hence, the only constraint is $\tilde{\phi} \tilde{h}=y$, which reduces the size of our problem.

We also develop our algorithm CS-OFDM for the purpose of handling noisy measurements. The iterative support detection (ISD) scheme proposed in [17] has a very good performance for solving (5) even with noisy measurements. Our algorithm uses the ISD, as well as a final denoising step. In the main iterative loop, it estimates a support set $I$ from the current reconstruction and reconstructs a new candidate solution by solving the minimization problem $\min \left\{\sum_{i \in I^{c}}\left|\tilde{h}_{i}\right|: \tilde{\phi} \tilde{h}=y\right\}$, and it iterates these two steps for a small number of iterations. The idea of iteratively updating the index set $I$ helps catch missing spikes and erase fake spikes. This is an $\ell_{1}$-based method but outperforms $\ell_{1}$. Analysis and numerical performance of ISD can be found in [18]. Because the measurements have noise, reconstruction is never exact. Our algorithm uses a

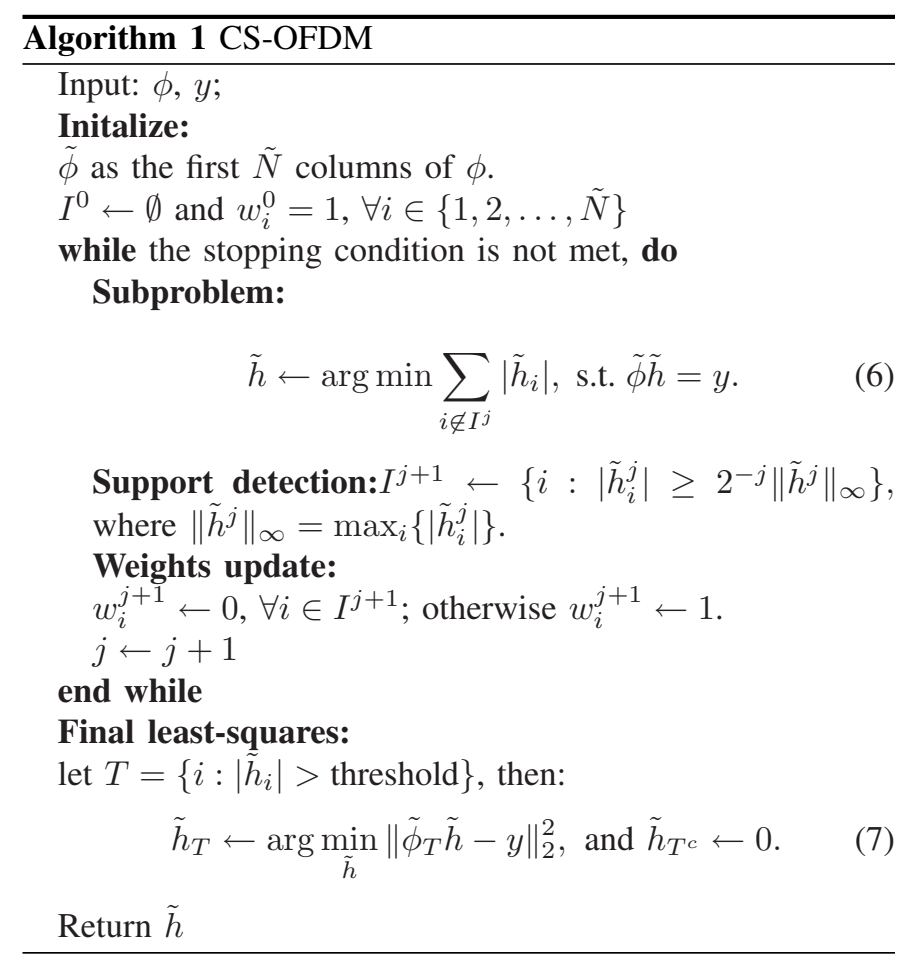

final denoising step, which solves least-squares over the final support $T$, to eliminate tiny spikes likely due to noise. Our pseudocode is listed in Algorithm 1.

In Algorithm 1, at each iteration $j$, (6) solves a weighted $\ell_{1}$ problem, and the solution $h^{j}$ is used for support detection to generate a new $I^{j+1}$. After the main loop is done, a support $T$ is estimated above a threshold, which is selected based on empirical experiences. If the support detection is executed successfully, $T$ would be the set of all channel multipath delay. Finally, $\tilde{h}$ is constructed by solving a small least-squares problem, and $\tilde{h}_{i}, \forall i \notin T$ fall to zero.

\section{Complexity Analysis}

This algorithm is efficient since every step is simple and the total number of iterations needed is small. The subproblem is a standard weighted $\ell_{1}$ minimization problem, which can be solved by various $\ell_{1}$ solvers. Since $\phi$ is a convolution operator, we choose YALL1 [19] since (i) it allows us to customize the operators involving $\tilde{\phi}$ and its adjoint to take advantages of DFTs, making it easier to implement the algorithm on hardware, (ii) YALL1 is asymptotically geometrically convergent and efficient even when the measurements are noisy. With our customization, all YALL1 operations are either an DFT/IDFT or one dimensional vector operations, so the overall complexity is $O(N \log N)$. Moreover, for support detection, we run YALL1 with a more forgiving stopping tolerance and always restart it from the last step solution. Furthermore, YALL1 converges faster as the index $I^{j}$ gets closer to the true support. The total number of YALL1 calls is also small since the detect support threshold decays exponentially and bounded below by a positive number. Numerical experience shows that the total number of YALL1 calls never exceeds $P$. 


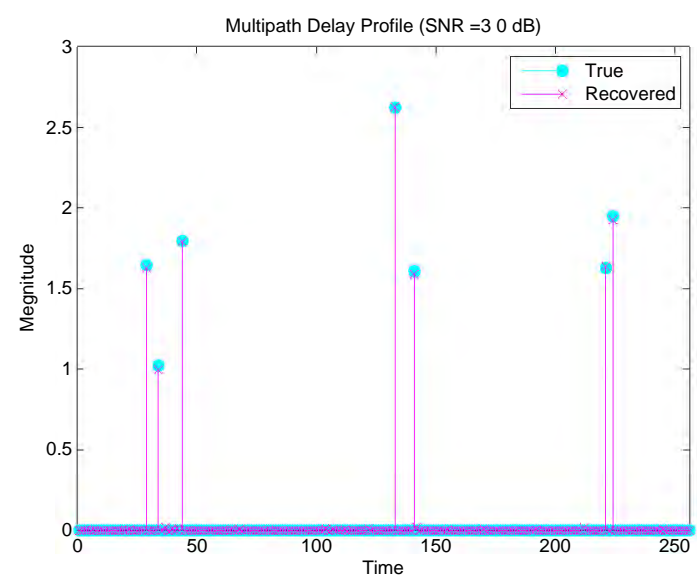

Fig. 3. Multipath Delay Profile.

The computational cost of the final least-squares step is negligible because the associated matrix $\tilde{\phi}_{T}$ has its number of columns approximately equal to the number of spikes in $h$, which is far less than its rows. For example, if the system has $P$ multipaths, the associated matrix for least-squares has size $M \times P$. Generally speaking, the complexity for this leastsquares is $O\left(M P+P^{3}\right)$. Since $P$ and $M$ are much smaller than $N$, the complexity of the entire algorithm is dominated by that of YALL1, which is $O(N \log N)$.

\section{Numerical Simulations}

In this section, we perform numerical simulations to illustrate the performance of the proposed CS-OFDM algorithm for high resolution OFDM channel estimation. Over varying channel profiles and SNR, we evaluate the the mean square errors (MSE) of channel estimation, as well as the performance of the multipath delay detection.

\section{A. Simulation Settings}

We consider an OFDM system with $1 k$-point IDFT ( $N=$ $1024)$ at the transmitter and 64-point DFT $(M=64)$ at the receiver, where we have a compression ratio of 16 . The number of silent sub-carrier which acts as guard band is 256 among 1024 sub-carriers. The channel is estimated based on 768 pilot tones with uniformly random phases and a unit amplitude, with the Gaussian noise level ranging from $10 \mathrm{~dB}$ to $30 \mathrm{~dB}$. We assume the usage of cyclic prefix and the impulse response of the channel is shorter than cyclic prefix which means there is no inter-symbol interference. For all simulations, we test the total numbers of multipath from 5 to 15 . Moreover, we use only one OFDM symbol, i.e. use all non-silent subcarriers only once to carry pilot signals. All reported performances will substantially improve if more pilots are inserted.

\section{B. MSE Performance}

Figure 3 is a snapshot of one channel estimation simulation. It suggests that the proposed pilot arrangement and CS-OFDM successfully detect an OFDM channel with 7 multipaths when the signal to noise ratio is $30 \mathrm{~dB}$. Our method not only exactly

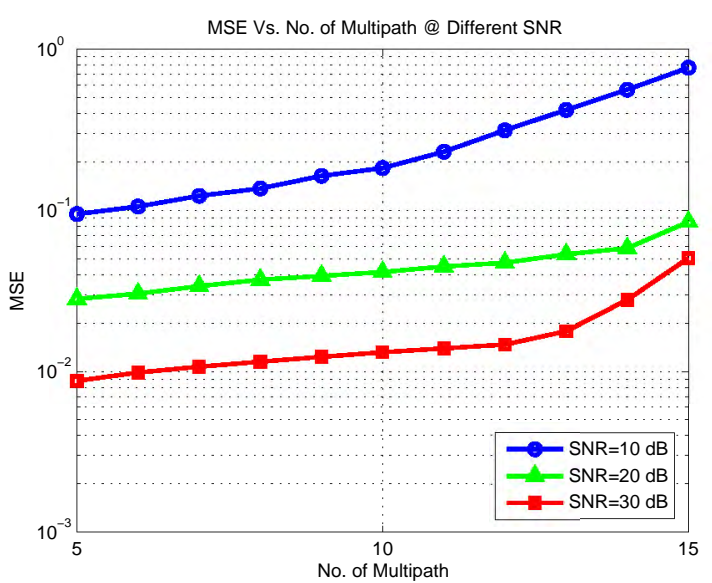

Fig. 4. MSE Performance.

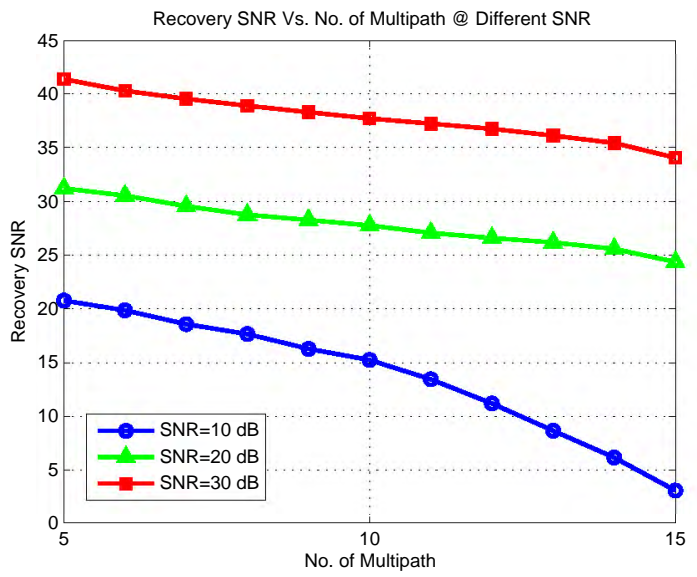

Fig. 5. Reconstructed SNR.

estimates the multipath delays but also correctly estimates the values of the corresponding multipath components.

Figure 4 depicts the MSE performance on OFDM channels with the number of multipaths ranging from 5 to 15 and noise level ranging from $10 \mathrm{~dB}$ to $30 \mathrm{~dB}$. When there are only a moderate number of multipaths on the OFDM channel, e.g. 10 multipaths, even when SNR is $20 \mathrm{~dB}$, MSE is as low as $-17 \mathrm{~dB}$.

Figure 5 shows the reconstructed SNR vs. the number of multipaths when the input SNR changes. We can see that CSOFDM achieves a gain in SNR. For example, when the input SNR is $10 \mathrm{~dB}$, we obtain a reconstructed SNR higher than 20 $\mathrm{dB}$ when there are 5 multipaths. As the number of multipaths increases, the SNR gain from the reconstructed signal to the input signal decreases. However, even when the number of multipaths is 10 , we still have a $5 \mathrm{~dB}$ gain, e.g. reconstructed SNR is $15 \mathrm{~dB}$ when the input signal SNR is $10 \mathrm{~dB}$. The similar SNR gain appears for input $\mathrm{SNR}=20 \mathrm{~dB}$ and $\mathrm{SNR}=30 \mathrm{~dB}$ cases. From the entire input SNR and the number of multipath range we have tested, there is an average gain of $6 \mathrm{~dB}$ from the input SNR to the recovered SNR. 


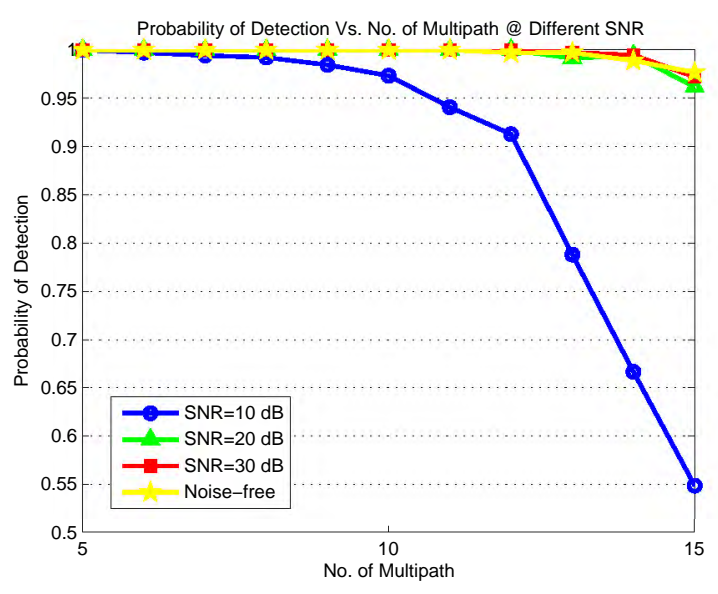

Fig. 6. Probability of Support Detection.

\section{Multipath Delay Detection Performance}

Figure 6 and Figure 7 depict the probability of correct detection (POD) of the multipath delay and the false alarm rate (FAR) while we change the SNR and the number of multipaths. When the SNR is above $10 \mathrm{~dB}$, simulation shows almost 100\% POD when the number of multipaths changing from 5 to 12 . When there is a relatively large number of multipaths, e.g. 15 , the probability of correct multipath delay detection is higher than $95 \%$ as $\mathrm{SNR} \geq 10 \mathrm{~dB}$. Even when SNR is low, as long as the number of multipaths does not exceed 10, we still have a POD of greater than $95 \%$. The FAR performance shows the similar results, as the SNR decreases and the number of multipaths increases, performance decreases. But, in a large range, e.g. SNR $\geq 10 \mathrm{~dB}$, the number of multipath $\leq 10$, we have almost zero FAR.

\section{CONCLUSiOnS}

Efficient OFDM channel estimation will drive OFDM to carry the future of wireless networking. An opportunity of high-efficiency OFDM channel estimation is given by the sparse nature of channel response. Riding on the recent development of sparse optimization and CS, we propose a design of probing pilots with random phases, which preserves the information of channel response during convolution and downsampling, and a sparse recovery algorithm, which returns the channel response in high SNR. These benefits translate to the high resolution of channel estimation, the lower speed of the receiver ADC, as well as shorter probing times. In this paper, the presentation is limited to an idealized OFDM model, intuitive explanations, and simulated experiments. In the future, we will formalize the work with rigorous theorems and fuse it into more realistic OFDM frameworks. The results presented here hint a noticeable improvement of OFDM performance in practice.

\section{REFERENCES}

[1] O. Edfors, M. Sandell, J.-J. Van de Beek, D. Landström, and F. Sjöberg, "An introduction to orthogonal frequency division multiplexing," Lulea Sweden: Luleả Tekniska Universitet, 1996, pp. 1-58.

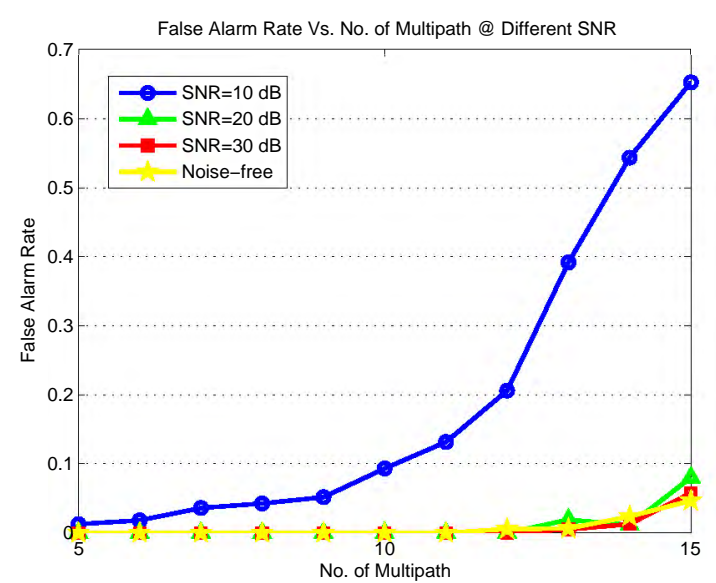

Fig. 7. Probability of False Support Detection.

[2] S. Takaoka and F. Adachi, "Pilot-assisted adaptive interpolation channel estimation for OFDM signal reception," in proceedings of Vehicular Technology Conference, vol.3, p.p. 1777-1781, May 2004.

[3] J. Byun and N. P. Natarajan, "Adaptive pilot utilization for OFDM channel estimation in a time varying channel," in proceedings of Wireless and Microwave Technology Conference, Clearwater, FL, pp. 1-5, April 2009.

[4] D. Donoho, "Compressed sensing," IEEE Trans. on Information Theory, vol. 52, no. 4, pp. 1289-1306, April 2006.

[5] E. Candes, J. Romberg, and T. Tao, "Stable signal recovery from incomplete and inaccurate measurements," Communications On Pure and Applied Mathematics, vol. 59, pp. 1207-1223.

[6] C.R. Berger, S. Zhou, P. Willett, B. Demissie, and J. Heckenbach, "Compressed sensing for OFDM/MIMO radar," in proceedings of the 42nd Annual Asilomar Conference on Signals, Systems and Computers, CA, pp.213-217, October 2008.

[7] C.R. Berger, S. Zhou, and P. Willett, "Signal extraction using compressed sensing for passive radar with OFDM signals," in proceedings of the 11th Int. Conf. on Information Fusion, Cologne, Germany, pp.1-6, July 2008.

[8] G. Tauböck and F. Hlawatsch, "A compressed sensing technique for OFDM channel estimation in mobile environments: exploiting channel sparsity for reducing pilots," in proceedings of IEEE Int. Conf. on Acoustics, Speech, and Signal Processing (ICASSP), Las Vegas, Nevada, pp.2885-2888, April 2008.

[9] C.R. Berger, S. Zhou, W. Chen, and Peter Willett, "Sparse channel estimation for OFDM: over-complete dictionaries and super-resolution methods," in proceedings of IEEE Intl. Workshop on Signal Process. Advances in Wireless Comm., Perugia, Italy, pp. 196-200, June 2009.

[10] E. Candes, J. Romberg, and T. Tao, "Robust uncertainty principles: exact signal reconstruction from highly incomplete frequency information," IEEE Trans. on Information Theory, vol. 52, no. 2, pp. 489-509, February 2006.

[11] E. Candes and T. Tao, "Near optimal signal recovery from random projections: universal encoding strategies," IEEE Trans. on Information Theory, vol. 52, no. 12, pp. 5406-5425, December 2006.

[12] Michel Ledoux, The Concentration of Measure Phenomenon, American Mathematical Society. ISBN 0821828649, 2001.

[13] W. U. Bajwa, J. D. Haupt, G. M. Raz, S. J. Wright, and R. D. Nowak, "Toeplitz-structures compressed sensing matrices," in proceedings of IEEE/SP 14th Workshop on Statistical Signal Processing, Madison, WI, pp. 294-298, August 2007.

[14] J. D. Haupt, W. U. Bajwa, G. M. Raz, and R. D. Nowak, "Toeplitz compressed sensing matrices with applications to sparse channel estimation," Submitted: August 29, 2008, Revised: March 17, 2010.

[15] J. Romberg, "Compressive sensing by random convolution," SIAM J. Imaging Sci., vol. 2, no. 4, pp. 1098-1128, November 2009.

[16] J. K. Romberg and R. Neelamani, "Sparse channel separation using random probes," to be submitted.

[17] Y. Wang and W. Yin, "Sparse signal reconstruction via iterative support detection," Rice University CAAM Technical Report TR09-30, 2009.

[18] W. Guo and W. Yin, "EdgeCS: edge guided compressive sensing reconstruction," Rice University, 2009.

[19] J. Yang and Y. Zhang, "Alternating direction algorithms for $\ell_{1}$-problems in compressives sensing," Rice CAAM Report TR09-37. 